\title{
Carnets
}

Revue électronique d'études françaises de l'APEF

Deuxième série - 14 | 2018

Études de génétique théâtrale et littéraire

\section{La « mise en pièce » de Renée : Genèse et impératifs de l'adaptation d'Émile Zola}

\section{Nejma Omari}

\section{(2) OpenEdition}

Journals

\section{Édition électronique}

URL : http://journals.openedition.org/carnets/8694

DOI : 10.4000/carnets.8694

ISSN : 1646-7698

Éditeur

APEF

\section{Référence électronique}

Nejma Omari, «La « mise en pièce » de Renée : Genèse et impératifs de l'adaptation d'Émile Zola », Carnets [En ligne], Deuxième série - 14 | 2018, mis en ligne le 30 novembre 2018, consulté le 01 mai 2019. URL : http://journals.openedition.org/carnets/8694; DOI : 10.4000/carnets.8694

Ce document a été généré automatiquement le 1 mai 2019.

\section{(c) (i) (8)}

Carnets est mis à disposition selon les termes de la licence Creative Commons - Atribution - Pas d'utilisation commerciale 4.0 International. 


\title{
La « mise en pièce » de Renée : Genèse et impératifs de l'adaptation d'Émile Zola
}

\author{
Nejma Omari
}

Personnellement, je regardais la mise à la scène du
roman comme une tentative grave et dangereuse.
Jamais je n'aurais risqué cette tentative moi-
même. Fatalement, lorsqu'on transporte un roman
au théâtre, on ne peut obtenir qu'une œuvre moins
complète, inférieure en intensité ; en un mot, on
gâte le livre, et c'est toujours là une besogne
mauvaise quand elle est faite par l'auteur lui-
même. (Zola, 1881:8)

1 Malgré ces affirmations péremptoires en exorde de la préface de l'adaptation théâtrale de L'Assommoir mise en scène par Busnach et Gastineau en 1879, Émile Zola s'essaye quelques années plus tard à cette «besogne mauvaise » qui consiste à mettre en pièce l'un de ses romans. Alors que la transposition de ses ouvrages pour le théâtre était d'ordinaire confiée à des carcassiers ${ }^{1}$, l'auteur des illustres Rougon-Macquart se décide, in fine, à signer de son seul nom Renée, drame en cinq actes tiré du roman parisien La Curée. Si la pièce est représentée pour la première fois le 16 avril 1887 sur les planches du théâtre du Vaudeville, l'histoire de sa création débute bien en amont, six ans plus tôt, lorsque Zola entreprend d'adapter le roman pour Sarah Bernhardt qui «s'était prise de passion pour La Curée [et] voyait dans le personnage de Renée un rôle superbe et à sa taille » (Zola, $1887: 1$ ). Afin de répondre aux demandes réitérées de «l'impératrice » du théâtre de son siècle - «Songez-vous à moi ? À quand ma pièce? » (Zola, ibid) — Zola puise aux sources du roman les principaux ingrédients de son intrigue, mais intègre également des données provenant de textes de types génériques différents. Parmi ces documents, la nouvelle Nantas, publiée dans Le Messager de l'Europe puis dans Le Voltaire en juillet 1879, inspire les types et caractères de la pièce, tout en augmentant son potentiel dramatique. L'influence 
de la nouvelle sur le drame est telle que Zola considère Renée comme étant «une combinaison de La Curée et de Nantas » (Zola, $1887: 2$ ).

2 Outre la pièce et ses hypotextes, l'auteur rédige deux autres variations sur la thématique de l'ascension sociale d'un homme fort et ambitieux: l'ébauche d'un drame tirée de Nantas (écrit selon toute probabilité après 1881) ainsi qu'un scénario intitulé Un homme à vendre (composé vers 1873). Cette répétition d'une même trame narrative de texte en texte est un phénomène isolé voire unique chez Zola, qui compose ce qui pourrait s'apparenter à une micro-série parallèle aux Rougon-Macquart. Ainsi, la multitude des genres mobilisés autour de l'écriture d'une même intrigue soulève certains questionnements : dans quelle mesure ces textes contribuent-ils à l'élaboration de la pièce? Quelles indications nous apportent-ils sur le processus de création théâtral zolien? Ou encore, quels sont les procédés de transposition dramatique utilisés par l'auteur?

3 Nous verrons tout d'abord que ces textes protéiformes, qu'ils soient achevés ou au stade pré-rédactionnel, forment un parcours transgénérique du roman au théâtre, révélant les différentes étapes de la genèse de Renée ainsi que les mécanismes de composition dramatique zoliens. Toutefois, la circulation d'un médium à l'autre implique de nombreuses transformations, en particulier dans le cas du théâtre où il est question de passer du logos à la leixis. S'il n'existe pas au XIX ${ }^{e}$ siècle de poétique légiférant le genre hybride de l'adaptation, il s'agit pour l'auteur d'opérer un travail de remodelage poétique de l'hypotexte en reformulant le roman de sorte à en obtenir une version condensée. Enfin, nous constaterons que ces métamorphoses sont également motivées par la volonté de satisfaire aux codes d'un médium gouverné par les ficelles et les recettes. Effectivement, les exigences et le goût du public concurrencent la souveraineté du génie défendue par l'auteur-théoricien dans ses campagnes naturalistes. L'exercice transmodalisateur engage alors un amalgame périlleux entre conventions et vérité essentielle des caractères et des tempéraments.

\section{Le dossier génétique de la pièce : les différentes étapes de la dramatisation de La Curée}

4 Au commencement, Zola créa les dossiers préparatoires au roman. Ce premier chapitre de la genèse de la pièce paraît particulièrement intéressant lorsqu'il s'agit d'étudier le processus de création dramatique amorcé par l'auteur, dans la mesure où il ne semble pas exister, à notre connaissance, de manuscrit de Renée qui nous permettrait d'examiner l'œuvre théâtrale «en gestation ». Il y a bien la préface du drame, dans laquelle Zola promet, dès la première phrase, l'histoire de la pièce - «Renée a une histoire que je désire conter avant tout. Elle me paraît curieuse et instructive » (Zola, 1887:1) - mais il s'agit davantage pour l'auteur d'éclaircir le contexte de production que de fournir des précisions quant à l'élaboration de l'œuvre dans ce récit préfaciel. A contrario, les dossiers préparatoires au roman semblent contenir en germe certains éléments constitutifs du drame.

Claude Duchet définit les dossiers préparatoires des œuvres de Zola comme «un ensemble instable, inachevé, parcellisé de matériaux rédactionnels ». C'est tout à fait exact, si l'on considère que travailler sur des manuscrits, sur des dossiers préparatoires, c'est travailler sur de l'inachevé, sur ce qui aurait pu être, sur ce qui a été à un moment de la rédaction, de la gestation du texte. (Leduc-Adine, 2002:8) 
En examinant les différents éléments de la pièce, nous pouvons constater que «ce qui aurait pu être » dans La Curée, se matérialise parfois concrètement dans Renée. En effet, bien que dix années s'écoulent entre les premiers plans du roman rédigés en 1869-1870 et la pièce que Zola écrit vers 1881, les manuscrits semblent constituer une sorte de " protohistoire » de l'adaptation. Nous pouvons aisément imaginer que Zola consulte luimême la centaine de feuillets de méditations sur l'œuvre lorsqu'il décide de l'adapter pour la scène. Dans les «Premiers détails» (qui se rapprochent de ce que l'auteur appellera ensuite l'«Ébauche»), Zola mène une réflexion sur le roman à venir en explorant divers scénarios et possibilités. Parmi les idées que l'auteur développe, émerge l'hypothèse d'un Aristide Saccard amoureux de sa femme: «[o]n pourrait peut-être introduire une étude physiologique. Aristide tombant amoureux fou de sa femme après un fait, ce qui n'est cependant pas dans la logique de son caractère » (Zola, 1871 : fo 297 r). Or, si le Saccard du roman, impitoyable spéculateur insensible à l'adultère commis par sa femme, parvient à tirer profit de l'inceste, celui de la pièce tombe finalement « amoureux fou » de Renée et décide de lui avouer ses sentiments :

Saccard.

Dites un mot, un seul... (Un silence; puis, ardemment, à demi-voix.) Renée! Oh!

Renée, je vous aime!

Renée, reculant.

Non! Non!

Saccard.

Je vous aime, Renée, je vous aime... Cela est venu je ne sais comment tout d'un coup. Oh! J'ai lutté, je me rappelais notre premier entretien, je trouvais cela indigne de moi... Mais, aujourd'hui, je souffre trop, il faut que je vous dise... (Zola, 1887 : 105).

6 Le sentimentalisme qui n'est pas « dans la logique » du caractère du Saccard de La Curée semble alors faire partie intégrante du tempérament du personnage de la pièce. Toutefois, avant de faire son apparition dans le drame, ce type du mari amoureux survient pour la première fois dans la nouvelle Nantas où le personnage éponyme finit par obtenir l'amour de Flavie lors d'un dénouement heureux, tandis que Renée préfère son beau-fils dans la pièce.

7 Outre la physionomie d'un Saccard sentimental, le motif du théâtre apparaît également dès les dossiers préparatoires et s'insère dans le roman à venir, avant même qu'il ne soit roman. Effectivement, en commentant son premier "plan », l'auteur émet l'idée d'une représentation théâtrale à laquelle Renée, qui se nommait alors Blanche, assisterait avec son beau-fils et amant : « Renée à une représentation de Phèdre, avec Maxime 'Ces anciens prennaient [sic] les choses tragiquement'« (Zola, 1871: fo241 r). Dans le roman, ce spectacle fonctionne comme un véritable révélateur qui incite Renée à se reconnaître dans le tempérament du personnage racinien. La «montée " du motif de Phèdre se poursuit ensuite du roman au théâtre et Renée évolue du stade de simple spectatrice au Théâtre-Italien à celui de protagoniste principal d'un drame en cinq actes. Dès lors, l'incitation personnelle de Zola, qui souhaite écrire « une nouvelle Phèdre » (Zola, ibid, fo 298 r), prend tout son sens avec la création de Renée, qui marque un retour aux sources classiques $^{2}$. Les manuscrits de l'hypotexte nous permettent donc de remonter aux origines premières de la création dramatique zolienne et ainsi d'observer les perspectives nouvelles qu'offre le médium théâtre.

8 Le deuxième chapitre de la genèse de la pièce débute au cours des mois qui suivent la publication de La Curée, lorsque Zola écrit une nouvelle variation autour de la thématique de l'homme fort sous la forme d'un scénario qu'il intitule Un homme à vendre. Ce récit de 
création de onze pages, au stade d'ébauche, ne sera jamais publié par l'auteur, bien que certains éléments aient été réutilisés lors de la composition de la nouvelle, puis de la pièce. Si l'intrigue du scénario était initialement sans rapport avec la trame narrative de La Curée, Zola intègre, au fil de la plume, des éléments qui rappellent le roman : « Voyons les personnages. [...] Un homme qui a épousé une femme pour son argent. Une sorte de courtière et d'entremetteuse, $\mathrm{M}^{\mathrm{me}}$ Sidonie » [...] L'affaire craque déjà. Le cabinet. La curée. Nouer solidement l'intrigue. La double intrigue » (Zola, 2009).

L'écrivain s'éloigne ensuite de La Curée afin de développer, quelques pages plus loin, des caractères que l'on retrouve dans les textes suivants : le portrait d'une dame fière, noble et méprisante et de son mari amoureux, tranchent alors avec la belle-mère incestueuse et le spéculateur que sont respectivement Renée et Saccard dans le roman. Le scénario, qui avait d'abord commencé comme une comédie, bascule peu à peu vers le drame - «C'est ici que le drame pourrait se nouer » - avec l'idée d'un acte supplémentaire : «Il faudrait un autre acte, qui pourrait encore agrandir l'idée. [...] il faudrait une complication aux actes 3 et 4 (la pièce étant en cinq actes.) [...] La complication pourrait venir de Renée ». Or, c'est bien avec Renée que « vient la complication » dans la pièce, lorsque celle-ci avoue son amour à Maxime à la dernière scène du troisième acte :

Maxime.

Qu'avais-tu donc, ce soir ? Pourquoi me forcer à aimer Ellen ?... Je la connaissais mal, et elle a été si charmante...

Renée, violemment.

Elle n'est plus là n'est-ce pas? Tu l'as mise à la porte... Jure que tu ne la reverras jamais, jamais !... Mon Dieu! J'ai cru que je pourrais, et je ne peux pas, je ne peux pas!... (dans un long sanglot.) Je t'aime! (Zola, 1887 : 95)

Ainsi ce scénario, qui constitue la première tentative avortée d'une représentation théâtrale inspirée du roman, est particulièrement instructif car il nous permet de nous introduire dans l'atelier de l'écrivain afin d'examiner les modalités de la création dramatique.
"Soliloque programmatif»? On s'aperçoit rapidement que ce soliloque est en réalité un polylogue, qu'il a là non pas UN Zola qui s'exprime et programme UN texte à venir, mais PLUSIEURS Zola parlant de plusieurs textes à PLUSIEURS interlocuteurs. S'expriment là d'abord, deux Zola principaux, qui occupent en permanence le texte : le Zola écrivant qui se donne à lui-même prospectivement des consignes d'écriture pour un texte à venir, futur virtuel [...] et le Zola qui évalue immédiatement et rétrospectivement la consigne elle- même ou ses premières mises en application concrètes, qui joue donc le rôle de premier lecteur. (Hamon, 2009: 20-21)

11 C'est effectivement sur le mode du polylogue que l'auteur crée cette série autour d'un même canevas : alors que la diversité générique multiplie les destinataires, les années qui séparent les différents textes impliquent, bien après la consigne, une interaction entre plusieurs Zola.

12 Si nous avons pu voir que les brouillons et les ébauches sont précieux lorsqu'il s'agit d'observer la germination de l'adaptation, la nouvelle Nantas apparaît comme une étape essentielle de la dramatisation du roman. Effectivement, pour qu'un drame tiré de La Curée devienne "possible, tout en restant fort dangereu[x] », il était nécessaire pour Zola d'esquiver l'inceste réel en trouvant l'expédient, la tricherie. Le fait que Saccard ne soit "que de nom le mari de Renée » rend la représentation moins risquée comme l'explique Zola dans la préface de la pièce. 
13 Ainsi, plus qu'une "combinaison entre La Curée et Nantas", Renée est un véritable compromis entre le roman et la nouvelle qui permet à l'auteur de rétablir dans la pièce les personnages de Maxime et de Louise (nommée Ellen dans Renée), tout en évitant l'inceste réel. Cette conciliation est particulièrement visible dans le cas du personnage de Saccard, qui semble souffrir d'un dédoublement de personnalité dû à sa double source : alors que le spéculateur tente d'escroquer sa femme en lui faisant signer un acte de vente, le mari amoureux essaye finalement de l'en dissuader au moment de conclure l'affaire :

Saccard, après un silence, la rappelant.

Renée, si vous me demandiez conseil, je vous empêcherais de conclure cette affaire.

Renée, revenant.

Pourquoi donc?

Saccard.

Parce qu'on vous vole, comme vous l'avez senti... Tenez! Voulez-vous que nous jetions cet acte au feu? (Zola, $1887: 104)$

14 Bien que le Saccard de la pièce perde de sa superbe, ce détour par la nouvelle semble nécessaire afin d'édulcorer l'adaptation.

Par ailleurs, le fort potentiel dramatique de Nantas en fait un « drame tout fait » comme le note Théodore de Banville. Effectivement, l'organisation du texte rappelle celle d'une pièce de théâtre : les deux premiers chapitres de la nouvelle, qui constituent l'exposition, présentent Nantas prêt au suicide après avoir vu ses tentatives d'ascension sociale réduites à néant; la fin du chapitre deux, quant à elle, amène l'élément perturbateur, l'attirance de Nantas pour Flavie, « très belle », et qui pourrait " le gêner dans la vie ». Les deux chapitres suivants développent alors la crise, qui atteint son paroxysme lorsque Nantas, pensant que sa femme a un amant, décide à nouveau de se suicider. Enfin, il s'agit dans le dernier chapitre du dénouement, complet, conformément aux règles dramatiques.

Les nombreuses indications concernant les déplacements et la gestuelle des personnages, qui s'apparentent à des didascalies kinésiques, ou encore les entrées et sorties quasi théâtrales qui séquencent le texte romanesque en scènes, participent également au caractère dramatique de la nouvelle. Rien d'étonnant donc à ce que Zola ait pensé à adapter Nantas pour le théâtre. En effet, l'auteur compose un plan de drame, non daté, qu'il intitule également du nom du personnage principal de la nouvelle. S'il n'y a pas d'indication précise quant à la rédaction de ce plan, Zola écrit pour lui-même: " $\mathrm{Au}$ deuxième acte, tout garder, excepté la scène de Renée et de Béraud, et encore voir cela ». Cette référence à la pièce écrite en 1881 est un indice de l'antériorité du manuscrit par rapport à l'écriture de Renée. Ainsi, bien que le plan de ce drame ne soit pas une étape de la dramatisation de La Curée, il révèle un peu plus une forme d'obsession de Zola pour cette figure de l'homme fort misérable et pauvre d'abord, puis au sommet de sa puissance ensuite, qui se répète inlassablement dans chacune de ces réécritures. L'auteur paraît effectivement poussé par un besoin interne de revenir aux données du roman La Curée. Cette sorte de logique inconsciente de la fiction est d'autant plus intrigante que le récit fictif semble résonner avec l'expérience marquante que fait le jeune Zola de la misère lorsqu'il quitte sa province aixoise pour la capitale. C'est alors un Zola « en puissance » qui s'écrit dans les répliques de son personnage qui s'écrie : «je suis une force !».

17 En définitive, le dossier génétique de la pièce, composé de textes de types variés, achevés ou à peine ébauchés, est fourni et hétérogène. Cette série de variations nous permet de repérer la circulation de motifs, de caractères, d'éléments d'intrigue ou encore de blocs textuels récurrents d'un médium à l'autre. Mais ce parcours transgénérique ne s'effectue 
pas sans certaines modifications liées aux spécificités de chaque forme, ce qui contraint Zola à une véritable recréation dont nous allons examiner les modalités.

\section{Les modalités de la (re)création théâtrale : espace, temps, personnages}

«Le théâtre est au corps ce que le roman est à l'âme: la démarcation des deux genres passe entre ces deux dimensions de la personne humaine, et l'adaptation doit travailler à leur délicate réconciliation» (Chelebourg, 1998: 319). Selon la formule poétique de Christian Chelebourg, l'adaptation théâtrale doit réussir la périlleuse mission de concilier les exigences génériques et esthétiques du roman et du théâtre. Il est donc question de produire une œuvre capable de "fusionner » les deux genres tout en respectant les impératifs de chacun, ce qui implique certaines modifications structurelles, spatiales ou encore temporelles. Dans le cas du passage du roman au théâtre, il s'agit de passer de la représentation mentale que se fait le lecteur, à la représentation visuelle destinée au spectateur. Dans cette optique, l'adaptateur doit opérer certains choix qui vont généralement dans le sens de la simplification sans toutefois être infidèle au texte-source. L'adaptation théâtrale, à mi-chemin entre imitation et création, ne peut effectivement en aucun cas s'affranchir de son hypotexte que le public du xix ${ }^{e}$ siècle s'attend à retrouver sur les planches. Qu'en est-il des choix opérés par Zola lorsqu'il met en pièce La Curée? L'auteur propose-t-il seulement une «version condensée » des avant-textes de la pièce, ou Renée est-elle une « belle infidèle »?

La première variation majeure lorsqu'il s'agit de passer du roman au théâtre concerne le rapport au temps et à la durée. Effectivement, si le temps du roman est, par excellence, le passé, celui du théâtre est le présent : temps fictionnel et temps réel ne faisant alors plus qu'un. Dans le drame de Zola, il arrive cependant que l'auteur utilise le passé afin de résumer des événements qu'il aurait été difficile de représenter sur scène du fait de la condensation de la pièce. Cette pratique est conforme au principe de création dramatique édicté par Racine dans la préface de Britannicus, et par Aristote avant lui : «[1]'une des règles du théâtre est de ne mettre en récit que les choses qui ne se peuvent passer en action » (Racine, 1670). L'adolescence de Saccard et sa rencontre fortuite avec $\mathrm{M}^{\text {lle }}$ Chuin, dont le spectateur prend connaissance par la médiation d'un récit au passé disséminé dans les échanges des protagonistes, illustrent notamment cet usage. La réplique du spéculateur s'inspire en effet de la narration de Nantas, tout en prenant en charge la fonction de l'analepse du chapitre deux de La Curée qui développe l'arrivée de Saccard à Paris :

Saccard.

Oui, nous étions trois frères, et notre mère avait de l'orgueil pour nous [...] Le dimanche, quand je me promenais seul, je me sentais du génie. [...] C'était le sentiment très net d'une intelligence et d'une volonté. J'avais une phrase favorite, je répétais toujours : «Je suis une force ! » et l'on riait, lorsqu'on me voyait avec ma mince redingote noire, craquée jusqu'aux épaules, et dont les manches remontaient au-dessus des poignets... (Zola, $1887: 47-48$ )

Et ainsi dans la nouvelle :

Il y avait, chez Nantas, une ambition entêtée de fortune, qu'il tenait de sa mère. C'était un garçon de décision prompte, de volonté froide. Tout jeune, il disait être une force. On avait souvent ri de lui, lorsqu'il s'oubliait à faire des confidences et à répéter sa phrase favorite: "Je suis une force", phrase qui devenait comique, 
quand on le voyait avec sa mince redingote noire, craquée aux épaules, et dont les manches lui remontaient au-dessus des poignets. (Zola, 2004a : 28)

Zola se doit donc de « confronter le temps de l'horloge et/ou le temps historique avec le temps psychique, vécu de la représentation » (Ubersfeld, 1996 : 159), ce qui l'oblige à faire certaines ellipses. L'enfance de Maxime par exemple, longuement développée dans le roman afin de peindre « l'homme-femme des sociétés pourries » (Zola, 1871: f fo367 r), est résumée en trois courtes phrases dans la pièce : «Je suis las des femmes. J'ai grandi dans leurs jupes. Toutes m'assomment» (Zola, $1887: 58)$. Cette réduction ne doit cependant pas s'opérer au détriment de l'intelligibilité de la pièce et des caractères. En effet, l'adaptateur doit éviter l'écueil de trop couper, au risque de rendre le drame lacunaire et d'empêcher ainsi le public de goûter les différentes machinations de l'intrigue. Zola, dans son désir de concision, admet avoir parfois manqué de clarté :

Ce qu'on peut me reprocher, c'est d'avoir été trop bref d'explications, dans la scène entre mademoiselle Chuin et Saccard. Dix lignes de plus, et tout s'éclairait. Je n'ai pas songé à les écrire, croyant le point de départ suffisamment posé et ayant avant tout le désir d'être sobre. (Zola, ibid: 17 -18)

Si le temps est contracté dans le roman, l'espace est également réduit afin de rendre la pièce représentable sur la scène d'un théâtre. Les dossiers préparatoires de La Curée, qui contiennent une série de plans de l'hôtel Saccard (Zola, 1871 : f 270 r, f 2171 r, f 272 r), sont précieux lorsqu'il s'agit de visualiser la sélection faite par Zola lors de la mise en spectacle du roman. L'auteur choisit une fois encore l'hôtel dans son drame, (à l'exception du premier acte qui se passe dans le cabinet de Béraud du Châtel) mais le "réagence " afin d'en faire un véritable décor de théâtre. La confrontation des manuscrits aux didascalies initiales de chaque acte de Renée nous permet d'obtenir une idée précise des travaux d'aménagement mis en œuvre par Zola :

Acte II : Le cabinet de Saccard, Pièce très riche; pas de bibliothèque et pas d'œuvres [sic] d'art. Un grand bureau à gauche. Un canapé et un fauteuil à droite, devant la cheminée. Porte au fond et portes latérales. [...] Acte III : Un jardin d'hiver. Serre superbe, pleine de grandes plantes vertes, éclairée par des lampes cachées dans les feuillages. À droite, la porte du cabinet de Saccard. À gauche, la porte du salon. Sièges et canapés rustiques. [...] Acte IV : Le boudoir de Renée. Un petit salon très luxueux. On y sent la femme frileuse, détraquée et de goût délicat. Au fond, la cheminée et la porte d'entrée. À droite, la porte de la chambre à coucher. À gauche : au premier plan, une porte; au second, une fenêtre. Une petite table. Une chaise longue. Des sièges. [...] Acte $\mathrm{v}:$ Un petit salon très riche, ouvrant par une large baie sur la serre. De hautes portières de tapisseries ferment cette baie. À gauche, une porte donnant dans l'appartement de Renée. À droite, une porte conduisant au grand salon. Un petit secrétaire. Un canapé. Des sièges.

Dès lors, nous pouvons constater que Zola concentre les principales pièces de l'hôtel Saccard sur un seul et même étage pour les besoins du médium, tout en s'inspirant probablement des plans du roman qui décrivent un agencement identique. L'adaptateur a conservé les pièces où se déroulent les actions de première importance à savoir la serre, dont la description s'étale sur deux pages dans le roman, les quartiers respectifs de Saccard et Renée ainsi que le petit salon.

Le personnel romanesque n'échappe pas à cette purge occasionnée par la recréation théâtrale. Zola passe effectivement de plus de trente personnages dans le roman, à seulement sept dans la nouvelle. Finalement, le drame compte dix protagonistes. L'auteur y a réintégré Maxime et Louise-Ellen, disparus dans Nantas, ainsi que Larsonneau, le prête-nom de Saccard dans La Curée. Avec l'ajout de ces trois personnages appartenant au 
roman-source, Zola réintroduit le thème de la spéculation ainsi que la relation incestueuse entre Maxime et sa belle-mère, bien que, comme nous l'avons évoqué, l'inceste réel soit « esquivé ». Malgré cette augmentation du nombre de personnages de la nouvelle à la pièce, la liste reste, pour des raisons évidentes, relativement concise en comparaison à celle du roman. La disparition d'un certain nombre de personnages secondaires entraîne ainsi certaines modifications dans le script de la pièce: la suppression de la tante Élisabeth par exemple oblige Béraud à régler lui-même les modalités financières de sa transaction avec Saccard. C'est également à lui que revient la tâche d'informer son beau-fils de l'existence des terrains.

L'autre difficulté de la transmodalisation théatrale du point de vue du personnel du roman concerne la reprise de personnages déjà connus du grand public. Les comédiens ont effectivement la responsabilité d'incarner les êtres de papier déjà visualisés mentalement par les lecteurs du roman. «Dans une adaptation, les acteurs sont moins là pour jouer la pièce que pour animer les personnages du roman. C'est à celui-ci qu'ils doivent fidélité; c'est dans ses pages qu'ils sont priés d'apprendre leur rôle» (Chelebourg, 1998: 315). Les revues théâtrales qui fleurissent dans les journaux aux lendemains des représentations nous permettent de mesurer le succès de l'interprétation des comédiens. En effet, si la réception de Renée est en demi-teinte, la critique s'accorde pour saluer le jeu des acteurs :

L'interprétation, elle, est excellente. $\mathrm{M}^{\text {Ile }}$ Brandès a trouvé dans le personnage énigmatique de Renée l'occasion d'une de ses plus belles créations. Elle a fait admirer toutes les ressources de son talent, dont la force et la souplesse n'ont jamais paru plus grandes. [...] La soirée a été pour elle triomphale. (BernardDèrosnes, Le Gil Blas : 18 avril 1887)

L'interprétation est excellente. [...] M. Raphaël Duflos n'a pas à regretter d'avoir quitté le Théâtre-Français. Son début de samedi lui assigne la première place au Vaudeville. Il a excellemment composé le rôle de Saccard [...] Mme Dayne-Grassot a rendu d'une manière expressive, avec une vérité et une mesure rares, le type de $\mathrm{M}^{\text {lle }}$ Chuin, la Macette de Régnier. M. Mayer a adroitement dessiné la silhouette du louche agent d'affaires Larsonneau. Paix aux autres. (Bauer, L'Écho de Paris : 19 avril 1887)

Malgré les nombreux aménagements engagés pour les besoins de la représentation, la pièce, à la manière d'un palimpseste, laisse apparaître les traces de ses avant-textes. L'hypotexte romanesque est notamment saillant dans le travail de mise en dialogue du récit. À titre d'exemple, l'ennui de Renée, véritable topos romanesque de la femme de trente ans au xix $x^{e}$ siècle, se traduit en des termes analogues dans La Curée et dans Renée: " [a]u bout d'un silence, elle répéta, avec l'accent d'une colère sourde : - Oh ! je m'ennuie, je m'ennuie à mourir » (Zola, 1999 : 44). La « colère sourde » de Renée devient alors « rage d'impuissance » chez son homologue scénique : «Renée. Oh! De tous et de tout !... Je m'ennuie, je m'ennuie à sangloter seule, le soir, la face dans mon oreiller, prise d'une rage d'impuissance... » (Zola, 1887 : 65).

Cependant, la ressemblance la plus frappante est celle de l'adaptation avec son autre avant-texte, Nantas. Lorsqu'il compose la pièce, Zola manie effectivement la colle et les ciseaux en tirant de la nouvelle de très nombreux éléments : il recopie à l'identique de multiples passages, réagence certains paragraphes, réutilise des éléments d'intrigue. En ce sens, la pièce, qui doit beaucoup à la nouvelle, devrait être considérée non pas comme une adaptation fidèle du deuxième volume des Rougon-Macquart, mais bien comme un assemblage méticuleux de La Curée et de Nantas. Cette précision quant à la double source 
de l'adaptation aurait peut-être permis à l'auteur d'éviter le désappointement d'un public escomptant assister à une reprise exacte de l'intrigue romanesque :

Ce qui m'a surtout frappé, dans le monceau de comptes [sic] rendus que j'ai lus d'une façon très attentive, c'est le désappointement des critiques, qui connaissant La Curée, se sont fâchés de ne pas retrouver le livre dans la pièce. Cela achèverait de me faire condamner tout drame tiré d'un roman. Chacun, l'autre soir, au vaudeville, était évidemment venu avec la pièce faite dans la tête: Renée était de ce tempérament, agissait de cette manière, de même que Saccard et Maxime avaient telle importance, jouaient tel rôle; et l'on s'imagine la débâcle, lorsque rien de ce qu'on attendait ne s'est réalisé. J'avais pourtant pris le soin d'appeler la pièce: Renée, ce qui me semblait indiquer suffisamment qu'il n'était plus question de $L a$ Curée. (Zola, $1887: 14$ )

Certes, cette précaution quant au changement de titre laisse suggérer quelque distance avec le roman, mais il n'empêche que le spectateur allant assister à une pièce signée par Zola et ayant pour titre le nom du personnage principal de La Curée peut légitimement s'attendre à une certaine fidélité à l'hypotexte romanesque. Plus encore lorsque les prospectus et articles chargés de la promotion de la pièce à venir annoncent une adaptation théâtrale du célèbre roman. Cette confusion que déplore l'auteur ne semble donc pas tout à fait accidentelle et Zola n'est pas sans savoir que l'histoire sulfureuse de «l'incestueuse des temps modernes » tirée du livre à scandale est bien plus vendeuse que celle de Nantas.

Il n'est donc pas aisé de trouver la juste mesure entre fidélité et innovation, condensation et précision, promotion et exactitude, roman et théâtre. À ces dilemmes d'ordre technique s'ajoute un impératif supplémentaire dans le cas spécifique de Zola. En effet, l'adaptateur aux multiples casquettes ne peut être véritablement dissocié du théoricien et il est tentant de lire Renée à la lumière des combats engagés par l'auteur pour le naturalisme au théâtre. Zola, contraint de composer avec les exigences d'un public habitué aux ficelles mélodramatiques, doit également rester fidèle à ses concepts théoriques. Dès lors, entre concessions aux recettes théâtrales du temps et esthétique naturaliste : Renée est-elle le manifeste pour un renouveau du théâtre que Zola appelle de ses vœux?

\section{L'accouplement de la carpe et du lapin : Renée, une pièce mixte}

30 Le 14 avril 1887, deux jours avant la première de Renée au Vaudeville, Zola affirme dans Le Matin: "Quand je me mis à écrire Renée, il y a six ans, je ne me préoccupais guère de formules dramatiques. J'écrivis une pièce, comme je la comprenais, et comme je la sentais voilà tout ».

Or, c'est précisément en 1880, au moment même où Zola écrit Renée, que débute sa campagne naturaliste avec la publication, chez Charpentier, d'un vaste cycle critique regroupant une sélection d'articles parus dans Le Messager de l'Europe, Le Bien Public ou encore Le Voltaire entre 1876 et 1880 . Après Le Roman expérimental, la campagne se poursuit en 1881 avec des ouvrages tels que Le Naturalisme au théâtre en février, Nos auteurs dramatiques en avril, ou encore Les Romanciers naturalistes en juin. Zola se préoccupe donc tout à fait de «formules dramatiques » au moment où il rédige son 
adaptation de La Curée, contrairement à ce qu'il affirme à la veille de la première de Renée dans ce qui ressemble à une sorte de captatio benevolentiae.

Par ailleurs, suite au triomphe de l'adaptation théâtrale de L'Assommoir, l'auteur Zola fait part à Busnach de sa volonté d' " affirmer par une œuvre intégrale signée de son nom seul la vérité esthétique de la formule naturaliste » (Best, 1986 : 133). Ce désir semble découler d'un constat que l'auteur livre dans la préface de la pièce de 1879 : «J'en suis arrivé à conclure que le public était plus mûr pour le naturalisme que je ne le croyais moi-même. Peut-être aurait-on pu risquer la pièce dans sa vérité entière, sans l'accommoder selon la recette mélodramatique des théâtres du boulevard ». Dès lors, pourquoi l'auteur ne se livre-t-il pas pleinement à cette expérimentation avec Renée ?

Pour comprendre les choix de Zola, il semble utile de revenir brièvement sur le contexte de création de la pièce. En effet, lorsque l'auteur décide enfin d'adapter La Curée pour la scène après les sollicitations de Sarah Bernhardt, l'actrice est sociétaire de la ComédieFrançaise depuis 1875. Mais alors qu'il travaille sur l'adaptation, elle démissionne avec éclat du Français et crée sa propre compagnie avec qui elle part en tournée à l'étranger. Dès lors, l'auteur, "tout déconfit avec [s]a pièce ", consulte M. Émile Perrin, administrateur général du Français, qui le met en garde contre les dangers de la pièce: «le public de la Comédie-Française ne supporterait pas la Phèdre moderne possédée par son fils, presque sous les yeux de son époux [...] je suis convaincu que la pièce ne passerait pas ». À son retour en France, Sarah Bernhardt, encore " plus épouvantée que M. Perrin » à la lecture de la pièce, la trouve également trop audacieuse et décline le rôle principal. Deux directeurs, M. Koning du Gymnase et $\mathrm{M}$. Porel de l'Odéon, refusèrent également Renée et la pièce resta alors près de six ans à dormir au fond d'un tiroir avant que MM. Deslandes et Carré, directeurs du Vaudeville, acceptent de la jouer malgré ses grands dangers. Ainsi, l'ironie veut que la pièce que Zola écrit en se conformant aux nombreuses attentes du public exigeant de la Comédie-Française, soit finalement jouée au Théâtre du Vaudeville, où les contraintes sont moindres. Dans la préface de Renée, le théoricien reconnaît avoir abâtardi la pièce « en vue d'une comédienne et d'un théâtre » : «si j'ai apporté certaines modifications au roman, je l'ai fait dans le vif désir d'être joué par la grande tragédienne sur notre première scène française, mon tort, si tort il y a, est d'avoir voulu être pratique » (Zola, $1887: 2)$.

Les ficelles du mélodrame sont effectivement apparentes dès la deuxième scène du premier acte lorsque Béraud du Châtel, seul avant l'entrée de Renée, fait part de sa tristesse au public.

Béraud, seul.

Mon Dieu! Mon Dieu !... Ah! Que de souffrance ! Que de honte !... (il est retombé dans son fauteuil, sanglotant, le visage couvert de ses mains. Mais il se calme, par un effort de volonté; et, lorsque Renée entre, muette, très pâle, il la reçoit d'un visage dur et fermé.) Approchez... Il faut que je vous parle. (Zola, ibid : 36 )

Ce monologue pathétique, tendant à conduire l'émotion à son comble, ainsi que la figure du père noble, véritable personnage-type du mélodrame, annoncent l'allégeance de la pièce aux recettes de son temps.

Toutefois, si Zola intègre les exigences d'un public habitué à la récurrence de motifs identiques et aux scènes toutes faites, il a le mérite de s'essayer au naturalisme, comme l'indique la formule de la pièce :

La fameuse formule serait celle-ci : l'homme physiologique, psychologique si vous voulez, déterminé par les milieux, étudié dans les fonctions totales de la vie; tout 
l'intérêt de la pièce, placé dans l'analyse des caractères, sentiments et passions; et, pour action, un fait unique et vrai, produit et subi par les personnages, mettant en branle leur humanité, jusqu'à l'extrême conclusion logique. (Zola, ibid : 23)

Dès le titre de la pièce, Zola va effectivement dans le sens de cette focalisation sur l'Homme et semble ainsi abandonner les dénonciations du régime au cœur du roman de l'or et de la chair. Les scènes dans lesquelles l'auteur peint la dépravation du Second Empire sont coupées, tout comme les personnages secondaires qui servaient à dénoncer le fonctionnement du régime impérial. Les hommes d'affaires véreux, les politiques corrompus, les femmes du monde qui s'aiment d'amours homosexuelles: tous ont été supprimés par Zola dans le processus de transposition. S'il est, à de rares reprises, question de l'immoralité du Second Empire, c'est uniquement pour justifier le détraquement de Renée, véritable transfuge sous l'influence d'une société viciée. En ce sens, il semble que l'adaptation soit davantage dévouée aux théories naturalistes zoliennes que le roman original où « infidèle déjà à son projet naturaliste, Zola fait moins [...] une étude des personnages placés dans un milieu que l'étude d'un milieu placé dans des personnages » (Duchet, $1970: 21$ ).

Outre ce déterminisme social, la jeune femme est également sous l'influence des circonstances ambiantes. C'est effectivement dans l'«air brûlant » de la serre que Renée, " détraquée ", « les yeux allumés de fièvre » fait des rêves « de jouissances inconnues » et succombe à son désir incestueux envers Maxime, malgré les mises en garde du jeune homme : « il ne faut pas rester ici, l'odeur de ces plantes te rend folle».

Les lois de l'hérédité, pierre angulaire des Rougon-Macquart, sont également respectées dans la pièce où Renée apparaît tiraillée entre le sens moral hérité de son père et son attirance pour la dépravation, atavisme de sa mère dont nous apprenons le passé tumultueux. Zola retourne donc aux sources de ses inspirations en empruntant au Traité de l'hérédité naturelle de Lucas ou encore à Deschanel qui observe « les influences du sang et de la parenté » (Deschanel, $1864: 7$ ). Les naturalistes ne sont cependant pas les seuls inspirateurs de l'auteur qui prône, dans ses écrits théoriques, un retour aux classiques: «[a]ujourd'hui, nos classiques si dédaignés sont la seule source où l'on doit remonter, si l'on veut tenter une renaissance dramatique. Je le répète, il faut leur prendre leur esprit, et non leur formule» (Zola, 2004d: 252). Le motif de Phèdre confirme la volonté de l'auteur de prolonger la formule classique en y injectant les éléments de la doctrine naturaliste ${ }^{3}$.

Il apparaît ainsi que Renée, véritable modus vivendi entre convention et vérité, se situe à la croisée de plusieurs esthétiques. Si l'auteur tente de mettre en pratique ses principes naturalistes, il reste bridé par les impératifs du médium et l'horizon d'attente d'un public familier des mélodrames du Boulevard du crime. La souveraineté du public, «théorie bouffonne » selon Zola, n'en reste pas moins indiscutable au théâtre. En effet, pour que l'exercice transmodalisateur soit gage de succès et de fortune, il est nécessaire que la pièce soit acceptée par un théâtre et ne «tombe " pas le soir de la première. Ce processus de recréation, loin d'être récréatif, demande à l'écrivain un travail fastidieux de réécriture qui se précise au fil des avatars. En définitive et malgré les mauvais présages des diverses personnalités à qui Zola soumet le manuscrit, la pièce tient l'affiche et évite " une lourde chute » : «Renée a été jouée trente-huit fois et a produit une recette totale de soixante-douze mille huit cent dix-sept francs, ce qui met la moyenne à dix-neuf cent seize francs vingt-trois centimes» (Zola, $1887:$ 13), souligne Zola dans la préface de la pièce. 
« Au travail maintenant, et tâchons de mieux faire » (Zola, ibid : 32).

\section{BIBLIOGRAPHIE}

ACHER, Lionel (2008). « La Boucle transgénérique de Phèdre chez Zola » in Guéret-Laferté et Mortier (dirs). D'un genre littéraire à l'autre. Mont-Saint-Aignan : Universités de Rouen et du Havre. BECKER, Colette (1992). Lire le réalisme et le naturalisme. Paris : Dunod.

BECKER, Colette, LEDUC-ADINE, Jean-Pierre \& MITTERAND, Henri (1987). Genèse, structure et style de « La Curée ». Paris : SEDES.

BEST, Janice (1986). Expérimentation et adaptation, Essai sur la méthode naturaliste d'Émile Zola. Paris : José Corti.

CAPITANIO, Sarah (1994). «L'Hypertextualité chez Zola : le cas de La Curée », Les Cahiers naturalistes, $\mathrm{XL}, \mathrm{n}^{\circ} 68$, pp. 49-62.

CHELEBourG, Christian (1998). « Splendeurs et misères de l'adaptation. Pièce conférence en trois actes et six tableaux. Représentée pour la première fois dans le château de Cerisy-la-Salle le lundi 17 août 1998. ", in Montaclair Florent (dir.). L'Adaptation du roman-feuilleton au théâtre. Besançon : Presses du Centre.

DESChAnel, Paul (1864). La Physiologie des écrivains et des artistes ou Essai de la critique naturelle. Paris : Hachette.

ECO, Umberto (1993). De Superman au surhomme. Paris : Grasset \& Fasquelle.

DELEUZE, Gilles (1993). Différence et répétition. Paris : PUF.

DEZALAY, Auguste (1971). « La 'Nouvelle Phèdre' de Zola ou Les mésaventures d'un personnage tragique ", Travaux de Linguistique et de Littérature, IX, $\mathrm{n}^{\circ}$ 2, pp. 121-134.

DEZALAY, Auguste (1983). L'Opéra des Rougon-Macquart : essai de rythmologie romanesque. Paris : Klincksieck.

DEZALAY, Auguste (1990). « Le Dix-septième siècle de Zola ", Travaux de Littérature offerts en hommage à Noémi Hepp, Adirel, p. 180 et ss.

DUBoIs, Jacques (2000). Les Romanciers du réel, De Balzac à Simenon. Paris : Seuil.

DUCHET, Claude (1979). « Préface » de La Curée. Paris : Garnier-Flammarion.

GENETTE, Gérard (1982). Palimpsestes. La littérature au deuxième degré. Paris : Seuil.

GRÉSILLON, Almuth (1994). Éléments de critique génétique. Lire les manuscrits modernes. Paris : PUF.

HAMON, Philippe (1998). Le Personnel du roman, le système des personnages dans « Les RougonMacquart » d'Émile Zola. Genève : Droz.

HAMON, Philippe (2009). Le Signe et la consigne : essai sur la genèse de l'œuvre en régime naturaliste. Genève : Droz. 
LEDUC-ADINE, Jean-Pierre (2002). Zola, Genèse de l'œuvre. Paris : CNRS.

MITTERAND, Henri (1987). Le Regard et le signe. Poétique du roman réaliste et naturaliste. Paris :

PUF.

UBERSFELD, Anne (1996). Lire le Théâtre I. Paris : Belin.

VIVEIRos (DE), Geneviève (2008). « Renée. Une interview inédite de Zola », Les Cahiers naturalistes, LIV, $\mathrm{n}^{\circ} 82$, pp. 289-300.

ZOLA, Émile (2009). Un homme à vendre (vers 1873). Paris : Nouveau monde.

zoLA, Émile (2004a). Nantas suivi de Madame Sourdis (1878). Paris : LGF.

zoLA, Émile (2004b). Le Roman expérimental (1880). Paris : Nouveau monde.

ZOLA, Émile (2004c). Le Naturalisme au théâtre (1881). Paris : Nouveau monde.

zOLA, Émile (2004d). Nos auteurs dramatiques (1881). Paris : Nouveau monde.

zOLA, Émile (1999). La Curée (1871). Paris : Gallimard.

zOLA, Émile (1971). Nantas (plan de drame), publié par FRICHET-RECHOU, Jacqueline dans « 'Nantas' : de la nouvelle au drame ", Les Cahiers naturalistes, XVII, $\mathrm{n}^{\circ}$ 41, p. 23.

zOLA, Émile (1887). Renée, pièce en cinq actes. Paris : Charpentier.

zoLA, Émile (1887). « Une conversation avec Émile Zola sur sa nouvelle pièce », Le Matin.

zoLA, Émile (1887). « 'Renée' et la critique », Le Figaro.

zoLA, Émile (1881). «Préface de L’Assommoir au théâtre ». Paris : Charpentier.

\section{NOTES}

1. Nous pouvons recenser treize adaptations produites et représentées à Paris à partir des œuvres de Zola entre 1873 et 1902. Ces pièces sont le plus souvent produites en collaboration avec des hommes de théâtre habitués aux recettes, comme c'est le cas de L'Assommoir (1879), Nana (1881), Pot Bouille (1882), Le Ventre de Paris (1887) ou encore Germinal (1888). Il est difficile de mesurer la part prise par Zola dans ces adaptations presque toutes réalisées par le célèbre dramaturge William Busnach.

2. À ce sujet, voir Acher (2008: 249).

3. Il existe plusieurs travaux étudiant le motif de Phèdre chez Zola et l'influence des dramaturges du Grand siècle. À ce sujet voir Acher, 2008 ; Dezalay, 1971 ; Dezalay, 1990.

\section{RÉSUMÉS}

Majoritairement reconnu pour son engagement journalistique et ses romans, Émile Zola fait également partie des auteurs qui succombent à ce que Philippe Chardin nomme "la tentation théâtrale des romanciers ». Outre ses créations dramatiques originales, l'auteur adapte certains de ses romans pour la scène à la fin du XIX ${ }^{\mathrm{e}}$ siècle. C'est notamment le cas du drame en cinq actes 
tiré de La Curée (1871) et intitulé Renée, dont il semble particulièrement intéressant d'examiner la genèse. En effet, durant les dix années qui s'écoulent entre le roman et son adaptation à la scène, Zola compose trois autres textes également inspirés du deuxième opus des Rougon-Macquart : une nouvelle, Nantas ; le plan d'un drame tiré de cette nouvelle ; de même qu'un scénario ayant pour titre Un homme à vendre. Ainsi, il semble que La Curée et ses hypertextes forment une sorte de micro-série qui nous pousse à nous interroger sur la pratique de la transmodalisation dramatique et ses impératifs.

Usually known due to his journalistic commitment and his famous novels, Emile Zola succumbs to the «theatrical temptation » described by Philippe Chardin. Besides his original theatre plays, the author adapts some of his novels for the stage at the end of the nineteenth century. Such is the case of the drama in five acts adapted from La Curée and entitled Renée, which it's interesting to examine the creating process. Indeed, during the ten years between the novel and its adaptation, Zola writes three other texts inspired by Les Rougon-Macquart: a short story, Nantas; the plan of a drama based on this short story and a storyline entitled Un homme à vendre. Therefore, it appears that La Curée and its variations constitute a short serie which raises the questions of theatrical adaptation and its practical needs.

\section{INDEX}

Mots-clés : Zola (Émile), adaptation, théâtre, transgénéricité, sérialité

Keywords : Zola (Émile), adaptation, theatre, literary genre, seriality

\section{AUTEUR}

\section{NEJMA OMARI}

Paul Valéry Montpellier III nejmaomari [at]gmail.com 